\title{
Localization in Wireless Sensor Network Using a Mobile Anchor in Obstacle Environment
}

\author{
Sayyed Majid Mazinani and Fatemeh Farnia
}

\begin{abstract}
This paper presents localization in wireless sensor networks (WSNs) in presence of obstacles using a mobile anchor. The proposed scheme uses a mobile anchor, which finds its location via global positioning system (GPS). The mobile anchor broadcasts its coordinate while it navigates through sensing region. The static sensor nodes receive beacon messages when the mobile anchor and sensor nodes have overlapping signals. The sensor nodes process the beacon messages to find their own coordinates using location of the anchor. The anchor moves in a snake-like method, which consumes less energy compare to the other approaches such as random methods. In this method the need for specific ranging hardware is eliminated on the sensor nodes. This elimination voids the communication requirement between sensor nodes. Further, the presence of obstacles in the environment has almost no effect on the output of the system causes the obstacle can be detected by the mobile anchor. In their project, Castalia simulator is used for the simulation and validation purpose. The output of the simulation results of localization problem confirm less localization error and less energy consumption over other range free solution using mobile anchor considering an extensive variety of network conditions.
\end{abstract}

Index Terms-Localization, mobile anchor nodes, wireless sensor networks, obstacle environment.

\section{INTRODUCTION}

Localization wireless sensor network has been point of interest in recent years in many applications. With the ongoing revolution in the hardware and wireless technologies, wireless sensor network is becoming the most preferred option for many applications, e.g., environment monitoring, health care, wildlife habitat monitoring, and tracking of objects [1]. Sensor nodes are small devices, which equipped with functions for environmental sensing and wireless ability. A set of sensor nodes can create a wireless sensor network. Localization problem of these sensors is an interested area in WSNs due to its crucial information. In localization problem the researcher needs to find accurately the location of individual sensor.

In range-free scheme for localization, which is an interested scheme for many applications of WSNs, the accuracy is lower compare to the rage-based scheme but in comparison the cost of the hardware is lower than the one in range-based [2].

This study proposes a range-free scheme for locating static

Manuscript received January 7, 2013; revised April 16, 2013.

Sayyed Majid Mazinani is with the Iranian Construction Engineering Organization Province of Khorasan Razavi and Department of Electrical Engineering, Imam Reza University Mashhad-Iran (e-mail: Mazinani@ieee.org).

Fatemeh Farina is with the Department of Computer Engineering of Azad University Mashhad-Iran (e-mail: Fatemehfarnia@gmail.com). sensors by using a mobile anchor in presence of obstacles. The static sensors are in a two-dimensional coordinate system, which are distributed in sensing region. A mobile anchor, which has a snake-like manner, can navigate through entire regain. The strength point of this research is that the anchor has ability to face the obstacles and circulate them while cover the entire surface visiting only once every place passing any place more than once. The purpose of the research mostly focuses the less error of detecting the location and lower energy consumption of mobile anchor.

The remaining of the paper proceeds as follows. Section II presents the literature review of localization scheme. The proposed methodology of localization is presented in Section III. Next, Section IV presents the simulation and discusses the results of simulation. Finally, Section V concludes and presents the limitations of this research.

\section{LITERATURE REVIEW}

Localization in wireless sensor network has been interested in different kind of situations. Accuracy, cost, and scalability are three main factors to be considered when designing localization method with high energy efficiency. Table 1 shows the brief review of the literature in localization problem of wireless sensor network having different conditions.

[3] develops a Range-Free localization technique with mobile anchors equipped with GPS. Their method offers coarse positioning accuracy with low computational expense. Conversely the energy consumption of their scheme as the mobile anchors moves randomly.

Kim et al. developed a new range-based localization scheme of mobile beacon-assisted localization (MBAL) [4]. Their technique has simple computational algorithm for movement path selection. Their scheme also uses a new range-checking technique for position-ambiguity problem. Although this scheme offers more promising results over random movement method, it fails to have the lowest energy consumption then compare to other schemes.

Li et al. used graph theory for the path planning of mobile anchors [5]. They considered the wireless network sensor as a connected undirected graph; then they redefined the path-planning problem into traversing graph and Spanning Tree with Breadth-First (BRF) and Backtracking Greedy (BTG) algorithms for the latter.

Karim et al. developed a Range-free localizing scheme by using Mobile Anchor (RELMA) for large scale WSNs, offering higher accuracy and energy efficiency. However the movement of the mobile anchors is random, leading to more energy consumption [6].

Chen et al. proposed a localization technique based on ring 
overlapping with reference and blind nodes [7]. The anchor sensor node can move snake-like for localization static sensor nodes. They implement Received Signal Strength Indicator(RSSI) values to localize the rings including reference and blind nodes. By overlapping the rings with each other and by utilizing the location of reference nodes, they implemented an algorithm to localize the blind nodes.

Ou presented a location scheme in WSN applying mobile anchors with four directional antennas (DIR) [3]. However the movement of mobile anchor in the simulation is straight line, which may path a certain place more than one time, which cause higher energy consumption.

One of the advantages of proposed simulation over other simulation is that it needs minimum two beacon messages for locating static sensor nodes, while in many proposed models the minimum number of messages is three. Lower transmitting information in simulation means less cost which is the priority in localization of WSNs.

\section{PROPOSED APPROACH}

In this section we explain the system environment of the localization problem in wireless sensor networks. Following the anchor mobile characteristics and the method of finding the location of sensor nodes are presented. The purpose of this research is to present a robust method for estimating the location of sensors using a mobile anchor with low energy consumption in obstacle environment.

Localization parameters of our method are beacon messages.

\section{A. System Environment}

In the simulation there are two types of sensors including static sensors nodes and a mobile anchor node. Static sensors nodes are randomly distributed in a two-dimensional coordinate system. The locations (states) of static sensors are unknown since they do not use GPS receiver. The mobile anchor node, which is equipped with an Omni directional antenna, employs a GPS receiver to discover their locations when they navigate over the sensing field. The anchor is able to receive a message from sensor nodes while traveling through region.

\section{B. Beacon Messages}

Localization parameters of our method are beacon messages. In the proposed model the anchor node broadcasts messages called beacon messaged while navigating in the sensing area. The beacon message contains time of messaging which is a sequential number of message and its coordinate in the system. The sequence number and its location the anchor changes while it moves.

TABLE I: COMPARISON OF RELATED WORKS IN LOCALIZATION SCHEME FOR WSNS USING MOBILE ANCHOR

\begin{tabular}{|c|c|c|c|c|c|c|c|}
\hline & Ssu & MBAL & $\begin{array}{l}\text { Path } \\
\text { planning }\end{array}$ & RELMA & $\begin{array}{l}\text { Ring } \\
\text { overlapping }\end{array}$ & $\begin{array}{l}\text { Directional } \\
\text { antenna }\end{array}$ & $\begin{array}{l}\text { Proposed } \\
\text { method }\end{array}$ \\
\hline Centralized /Distributed & & & & $\begin{array}{l}\text { distributed } \\
\text { central }\end{array}$ & & & \\
\hline Scalability & $\mathrm{x}$ & $\mathrm{x}$ & $\mathrm{x}$ & $\mathrm{x}$ & $\mathrm{x}$ & $\mathrm{x}$ & $\mathrm{x}$ \\
\hline Range based/free & Range free & Range based & Range free & Range free & Range free & Range free & Range free \\
\hline mobile anchor movement & Random & Path decision & Graph & Random & Snake-like & Straigh line & Snake-like \\
\hline environment obstacle & & & & & & $\mathrm{x}$ & \\
\hline Number of mobile anchors & 1 & 1 & 1 & $\mathrm{n}$ & 1 & $\mathrm{n}$ & 1 \\
\hline Neighbor-information-based & & $\mathrm{x}$ & & $\mathrm{x}$ & & & \\
\hline Directional antenna & & & & & & $\mathrm{x}$ & $\mathrm{x}$ \\
\hline Energy consumption & high & medium & low & high & medium & high & low \\
\hline $\begin{array}{l}\text { minimum number of } \\
\text { messeages for localization }\end{array}$ & 4 & 3 & 3 & 3 & 4 & 6 & 2 \\
\hline
\end{tabular}

\section{Movement}

It is essential to reduce the path of mobile anchor and to have full coverage in order to save the hardware cost of sensor nodes. Therefore we define the movement of the anchor sensor node in a snake-like method to deploy static sensor node. As we see in Fig. 1, it shows the two-dimensional coordination system of WSNs existing one obstacle. Anchor begin from the corner of the sensing field (starting point is $(0,0)$ ) to navigate through entire area moving from left to right, moving down, then moving from right to left, again moving down and so on. An obstacle can be circulated by the snake-like method, since the mobile anchor change its direction while it faces the obstacle. When the anchor changes its direction it stores the information of circulating point for future movements to have correct direction. The mobile anchor transmits beacons periodically while they are travelling to set the coordinate for each static sensor nodes in turn. Accordingly, sensor nodes can cover every area in the sensing field while they don't travel any area more than once. This reduces the energy consumption of the mobile anchor while passing the entire region.

\section{Location Estimation}

While mobile anchor broadcasting the beacons sequentially, the sensor nodes can receive the beacon messages when the anchor is in transition neighbor. Each sensor node is able to calculate its location using two beacon messages. We assume that the coordinates of two different locations of mobile anchor is $\left(x_{1}, y_{1}\right)$ and $\left(x_{2}, y_{2}\right)$. Each sensor node calculates its coordinate according to (1) and (2).

$$
\begin{aligned}
& \left(x-x_{1}\right)^{2}+\left(y-y_{1}\right)^{2}=r^{2} \\
& \left(x-x_{2}\right)^{2}+\left(y-y_{2}\right)^{2}=r^{2}
\end{aligned}
$$

Fig. 2 shows the location estimation of a sensor node. As we see it reflects two estimated locations for each sensor node. For finding the best solution the anchor uses the degree of antenna. 


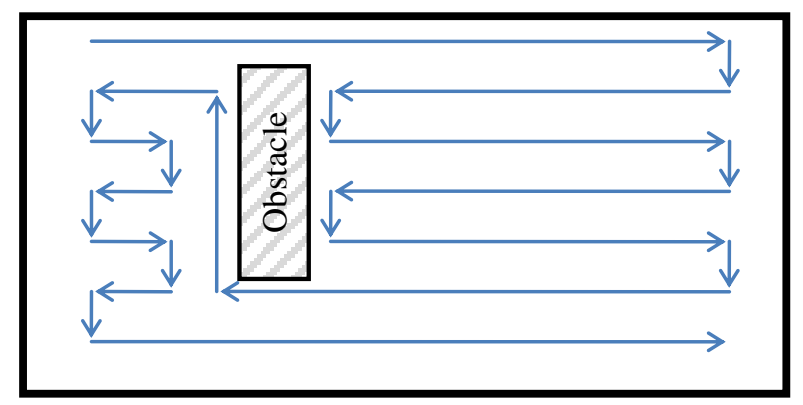

Fig. 1. Snake-like pattern of the mobile anchor facing an obstacle.

\section{Simulation AND ANALYSiS}

In this section the simulation environment, the performance of the proposed simulated model, and the results including the comparisons of different factors are analysed and presented.

\section{Simulation}

For simulating the proposed model, Castalia based on Omnet ++ is employed. In this simulation, the sensor region is considered as $10,000 \mathrm{~m}^{2}$ while there are 100 sensor nodes, which randomly distributed, and one mobile anchor. As we see in Fig.1, we define the initial position of the mobile anchor as top-left of the sensor filed. Using Snake-like pattern, it navigates through the entire region.

In our simulations, the average location error is the average distance between estimated location $\left(X_{e i}, Y_{e i}\right)$ and the actual location $\left(X_{i}, Y_{i}\right)$ of all sensor nodes. The average location error is defined in (3).

$$
\underline{\sum \sqrt{\left(x_{e i}-x_{i}\right)^{2}-\left(y_{e i}-y_{i}\right)^{2}}}
$$

We consider some parameters for showing the efficiency of the presented work. The simulation parameters are shown in Table II.

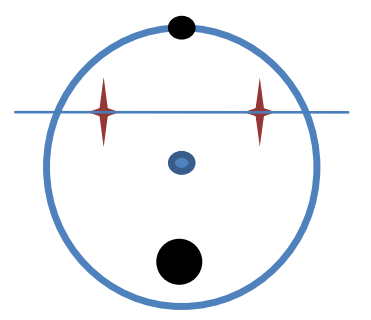

Anchor locations

Actual location of sensor node

Estimated locations

Fig. 2. Location estimations of a sensor node.

In our simulation we also consider the existence of obstacles as we also have this situation in real-word. Therefore we placed several obstacles in the sensing region to can have more realistic results.

TABLE II: SIMULATION PARAMETERS

\begin{tabular}{|c|c|}
\hline Parameters & Values \\
\hline $\begin{array}{c}\text { The impact of Beacon distance } \\
\text { on average localization error }\end{array}$ & $1,2,3,4,5 \mathrm{~m}$ \\
\hline $\begin{array}{c}\text { The impact of Beacon distance } \\
\text { on energy consumption }\end{array}$ & $1,2,3,4,5 \mathrm{~m}$ \\
\hline
\end{tabular}

\section{RESUlts}

In this section we consider some parameters to compare the results of this simulation to the other similar presented localization schemes. The most important factors to compare are average localization error and energy consumption of the anchor mobile.

\section{A. The Impact of Beacon Distance on Localization Error}

The impact of beacon intervals on the average localization error is a method to measure the efficiency of the presented localization scheme. To estimate the impact of beacon intervals, we implement simulations with different beacon intervals: $1 \mathrm{~m}, 2 \mathrm{~m}, 3 \mathrm{~m}, 4 \mathrm{~m}$ and $5 \mathrm{~m}$. We also compare our method with DIR method, which is more similar to our work. The simulation shows that the average localization error is more or less similar to the DIR method. However, we also compare the energy consumption of the anchor mobile of the two different methods.

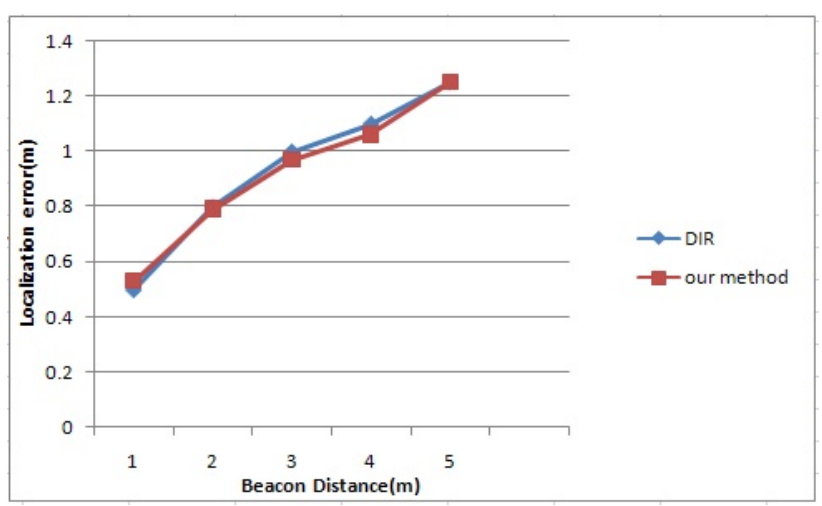

Fig. 3. Average localization error vs beacon distance.

\section{B. The Impact of Beacon Distance on Energy Consumption}

We also consider the energy consumption of the anchor mobile. For comparing the results with DIR method, we consider the impact of the bacon distance on the energy consumption. We consider $1 \mathrm{~m}, 2 \mathrm{~m}, 3 \mathrm{~m}, 4 \mathrm{~m}$ and $5 \mathrm{~m}$ as the beacon messages intervals. Fig. 4. shows that the energy consumption is lower in the presented method compare to DIR method.

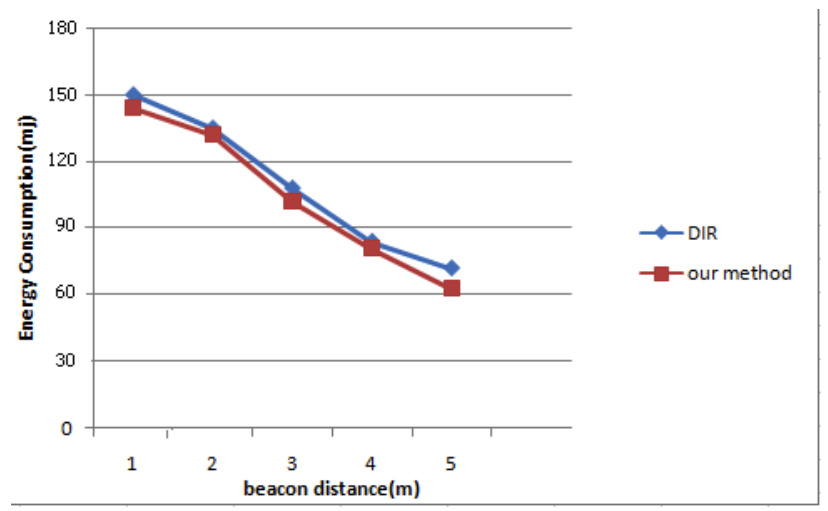

Fig. 4. Energy consumption vs beacon distance.

\section{CONCLUSION}

Localization problem is one of the important problems in many applications of Wireless Sensor Networks (WSNs). In addition, range free problems are also more applicable 
compare to range-based problems. The Simulation results show that the average localization of static nodes has less error compare to the other similar work. In our simulation we also have one anchor mobile for localization sensor nodes with minimum number of beacon transmissions, which cause lower cost. Energy consumption of the anchor also plays important role. We also consider this parameter to compare other similar works. The simulation results shows that it consume less energy using snake-like pattern in obstacle environment.

\section{REFERENCES}

[1] I. F. Akyildiz, W. Su, Y. Sankarasubramaniam, and E. Cayirci, "A survey on sensor networks," IEEE Communications Magazine, vol. 40, no. 8, pp. 102-114, August 2002.

[2] K. F. Ssu, C. H. Ou, and H. Jiau, "Localization with mobile anchor points in wireless sensor networks," IEEE Transactions on Vehicular Technology, vol. 54, pp. 1186-1197, May 2005.

[3] C. H. Ou, "A localization scheme for wireless sensor networks using mobile anchors with directional antennas," Sensors Journal, IEEE, vol. 11 , no. 7, pp. 1607-1616, 2011

[4] K. W. Kim and W. J. Lee, "MBAL: A mobile beacon-assisted localization scheme for wireless sensor networks," in Proc. 16th International Conference on Computer Communications and Networks, 2007. ICCCN 2007, IEEE, 2007.

[5] H. J. Li, J. W. Wang, X. Li, and H. X. Ma, "Real-Time path planning of mobile anchor node in localization for wireless sensor networks," in Proc. 2008. ICIA 2008. International Conference on Information and Automation, IEEE, pp. 384-389, 2008.

[6] L. Karim, N. Nasser, and T. E. Salti, "RELMA: A range free localization approach using mobile anchor node for wireless sensor networks," in Proc. Global Telecommunications Conference (GLOBECOM 2010), IEEE, 2010.

[7] Y. S. Chen et al., "An efficient localization scheme with ring overlapping by utilizing mobile anchors in wireless sensor networks," in Proc. 2010 4th International Conference on Multimedia and Ubiquitous Engineering (MUE), IEEE, 2010.

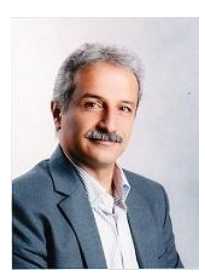

Sayyed Majid Mazinani was born in Mashhad, Iran on 28 January 1971. He received his Bachelor degree in Electronics from Ferdowsi University, Mashhad, Iran in 1994 and his Master degree in Remote Sensing and Image Processing from Tarbiat Modarres University, Tehran, Iran in 1997. He worked in IRIB from 1999 to 2004. He also received his phD in Wireless Sensor Networks from Ferdowsi University, Mashhad, Iran in 2009. He is currently assistant professor at the faculty of Engineering in Imam Reza University, Mashhad, Iran. He was the head of Department of Electrical and Computer Engineering from 2009 to 2012. His research interests include Computer Networks, Wireless Sensor Networks and Smart Grids.

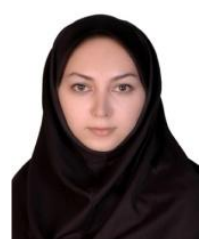

Fatemeh Farnia was born in Mashhad, Iran in Apri 1978. She received her bachelor degree in computer engineering from Azad university of Mashhad in 2010 where she is currently working toward M.S. degree in department of computer engineering. Her current research interests include wireless sensor networks, localization and mobile system. 